\title{
Biosensors Based on SOI Nanowire Transistors for Biomedicine and Virusology
}

\author{
O. V. Naumova ${ }^{a, *}$, V. M. Generalov ${ }^{b}$, E. G. Zaitseva ${ }^{a}$, A. V. Latyshev ${ }^{a}$, A. L. Aseev ${ }^{a, c}$, S. A. Pyankov ${ }^{b}$, \\ I. V. Kolosova ${ }^{b}$, G. G. Ananko ${ }^{b}$, A. P. Agafonov, E. V. Gavrilova ${ }^{b}$, R. A. Maksyutov ${ }^{b}$ and A. S. Safatov \\ ${ }^{a}$ Rzhanov Institute of Semiconductor Physics, Siberian Branch, Russian Academy of Sciences, Novosibirsk, 630090 Russia \\ ${ }^{b}$ Vector State Research Center of Virology and Biotechnology, Koltsovo, Novosibirsk region, 630559 Russia \\ ${ }^{c}$ Novosibirsk National Research State University, Novosibirsk, 630090 Russia \\ *e-mail: naumova@isp.nsc.ru
}

Received August 3, 2020; revised August 18, 2020; accepted November 18, 2020

\begin{abstract}
This article contains the results of research on the topical problem of highly sensitive express registration of biological objects using field-effect transistors with the surface open for analyte access, which are made based on silicon-on-insulator (SOI) films. The possibilities of dielectrophoretic effects for controlling the concentration of the analyte in the area of sensory elements are considered on the example of the indication of viruses of nuclear polyhedrosis and vaccinia. It is shown that the use of the dielectrophoresis (DEPh) effect makes it possible to solve (1) the key tasks for creating sensor systems: increasing the detecting ability, as well as exrtacting and verifying the signal from the target particles; and (2) the fundamental task: determining the charge state of the analyte in solutions without modifying the sensors' surface. The problems and prospects of the mass application of nanowire (NW) biosensors, including those with the dielectrophoretic effect, in biotechnology, virology, etc., are discussed.
\end{abstract}

DOI: $10.1134 / \mathrm{S} 1063739721030069$

\section{INTRODUCTION}

The recently aggravated problem of the emergence of an epidemic or pandemic is accompanied by new and increasingly higher requirements on the indication processes of biological and chemical analytes (viruses, pathogens, toxins, etc.), such as their sensitivity, specificity, and reliability of the results obtained; the speed of the procedure; performance, signal-to-noise ratio; power consumption; and cost. These circumstances require the development of new, more advanced technologies in relation to the existing and traditional options for solving this problem, based on the use of clinical, virological control methods, such as polymerase chain reaction, enzyme immunoassay, hemagglutination, analysis of pathogen morphology, and the use of laboratory animals.

In the past decade, the rapidly developing direction of the development of detectors based on nanowire (NW) sensors has been a competitive alternative to traditional analytical display systems. Such devices have unique characteristics in terms of sensitivity to biological molecules in the femtomole and subfemtomole ranges, rapidity, and the ability to display indicators in the form of electrical signals arising from the contact of analyte molecules with the surface of the sensor element. Signal digitization makes it possible to widely use the advantages of information technologies when processing and transmitting indication data through communication channels to a single information center, for an unlimited long-term storage of the indication results and computer statistical processing of an array of results and for achieving high reliability of the method.

In addition to the data collection and processing system, the components of the sensor devices are the analyte's delivery system to the sensor element; and a recognition element: a layer of receptors, enzymes, antibodies, DNA fragments, etc., (or the so-called layer of probes) on the surface of the sensor, which selectively interacts with the analyte-the object of research-and practically does not react with other substances in the solution/gas; and the sensory element itself, which converts the biochemical interaction of an analyte with a layer of probes on its surface into a measured signal [1]. In the past few decades, various solutions have been proposed/implemented for the creation of all components of sensor systems, including the formation technologies and material of the sensor element. Among the various types of NW sensors, a special place is occupied by silicon-based sensors (in particular, silicon-on-insulator (SOI) films). This is primarily due to the possibility of the mass production of sensors and diagnostic Lab-onChip systems using industrial silicon technology. 
Earlier at the Rzhanov Institute of Semiconductor Physics, Siberian Branch, Russian Academy of Sciences (ISP SB RAS) together with the Orekhovich Institute of Biomedical Chemistry, RAS, developed NW sensors with femtomolar $\left(10^{-15} \mathrm{M}\right)$ sensitivity to proteins, based on the so-called top-down technology, using optical and electron lithography of the nanoscale SOI layers. In [2-4], BSA bovine serum albumin molecules were used as the test molecules. To ensure the maximum response to the protein, the transistor characteristics of SOI sensors were used (the substrate of SOI structures was used as the control gate and a baried dielectric was used as a gate). This made it possible to provide internal signal amplification due to the exponential dependence of the current in the subthreshold SOI mode of the NW sensor (transistor). Subsequently, SOI-NW sensors were used to detect HBsAg markers of hepatitis B, the liver tumor marker $\alpha$-fetoprotein (sensitivity in the range of $10^{-14}$ to $10^{-15} \mathrm{M}$ ) [5], and the lung tumor marker D-NFATc1 with a sensitivity of $2.5 \times 10^{-15} \mathrm{M}[6]$.

The femtomole sensitivity level of NW sensors is limited by the probability of analyte adsorption on its surface. This limitation is partially removed, for example, by using microfluidic cells with the cyclic running of the test solution and/or by increasing the area of the sensor element. The approach we used made it possible not only to provide internal amplification of the readout signal but also to remove the limitation on the nanometer size of the sensor element.

The principle of operation of NW sensors is based on the field effect. The adsorption of particles leads to a change in the potential on the NW surface. Accordingly, the concentration of free carriers in the sensor and its conductivity change (in real time, which ensures the rapidity of the analyte's indication). If during adsorption, the sensor element is depleted by the free carriers in the local region and the depletion region is comparable to the dimensions of the NW element, then one particle is capable of completely blocking its conductivity. This results in the maximum response with extreme sensitivity: one particle per sensor element. Note that the experimental maximum sensitivity of NW sensors was achieved during the detection of the influenza virus in [7]. The width of the depletion region is determined by the concentration of the free carriers. The subthreshold transistor mode allows changing the concentration of the free carriers within several orders of magnitude, i.e., tuning the width of the depletion area. Accordingly, it becomes possible to remove the limitation on the nanometer size (increase the effective area) of the sensor element and move completely to optical lithography during its formation.

The femtomole sensitivity level for sensors with a micron width of the sensor elements (SOI strips) fabricated using the standard optical lithography was obtained by us by detecting short RNA sequences cor- responding to non-small cell lung cancer markers (with a dynamic range of 10 orders of magnitude) [8] and the subfemtomol level (up to $10^{-17} \mathrm{M}$ ) for micro-RNA contained in the blood plasma of patients with breast cancer [9]. The recent work [10] showed the possibility of using such sensors for the indication of the model protein VP-40 of the Ebola virus and its complexes with specific monoclonal antibodies.

A high degree of sensitivity of the sensors is achieved provided that the signal from the analyte is greater than the signal from the background particles. The signal from the background particles is determined by the response of sensors without probes on the surface. In other words, in order to verify the measurements, a chip with sensors must have at least two types of elements:

(1) modified with probes for selective specific interaction with the analyte;

(2) not modified or modified by nonspecific probes (for comparison). In [8], a method was proposed for modifying the surface of the sensors, which makes it possible to obtain its response to the background RNA (to the nonspecific interaction with the oligonucleotide probes), not more than $2 \%$, even when their concentration changes by 9 orders of magnitude (from $10^{-15}$ to $10^{-6} \mathrm{M}$ ). To suppress the background signal, we used the passivation of the residual bonds on the surface of the sensors that did not react with the probes with glycine molecules. The efficiency and features of the surface passivation of sensors were studied in [11].

At the same time, the prospects for the widespread use of SOI biosensors to realize their competitive advantages in solving practically important problems of modern biomedicine and virology are constrained by a number of problems. The main ones are listed below: (1) the problem of surface preparation and indication of the analyte in real biofluids with a high concentration of background particles of various types (for example, in blood, blood plasma with a particle concentration of $150 \mathrm{mM}$, nutrient media for viruses, and bacteria with a high concentration of proteins); (2) the problem of combining the technology of modifying the surface of the sensors with the formation of microfluidic cells; and (3) the problem of the accumulation of charge on the surface of the sensors during their storage [12]. The femtomole and subfemtomole sensitivity of the sensors, as mentioned above, is limited by the delivery of the analyte to the sensory elements. Dielectrophoresis (DEPh) is a promising solution to these problems.

$\mathrm{DEPh}$ is the electrokinetic movement of dielectrically polarized materials in an inhomogeneous alternating electric field [13]. The DEPh manipulation of bioparticles depends on their polarizability and the polarizability of the environment. The force acting in an inhomogeneous electric field of strength $E$ on a spherical particle with radius $r$ is defined as 
(a)

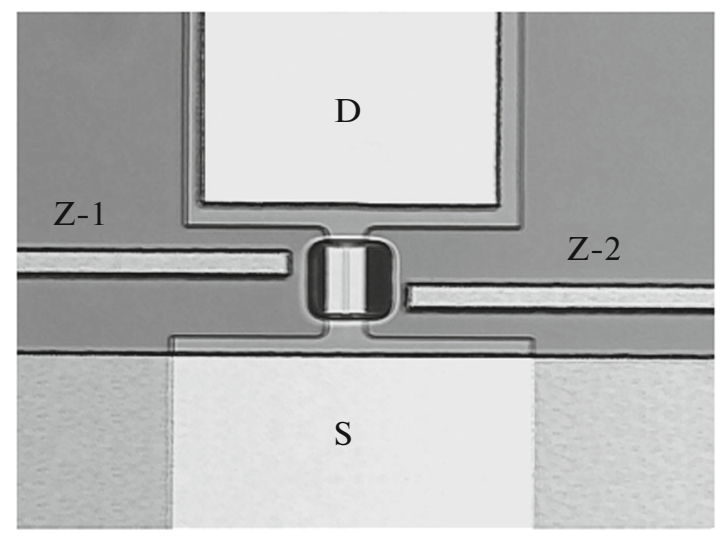

(b)

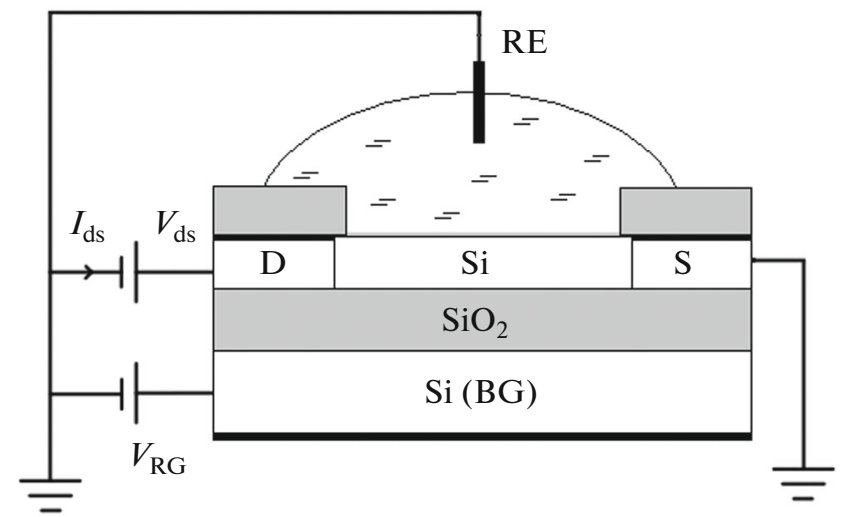

Fig. 1. Optical (a) and schematic (b) images of an SOI sensor: S, source; D, drain; Z-1 and Z-2, lateral DEPh-electrodes; RE, reference electrode.

$$
\begin{gathered}
\vec{F}=2 \pi r^{3} \varepsilon_{m} \operatorname{Re}\left[F_{C M}\right] \nabla\left|E^{2}\right| \\
=2 \pi r^{3} \varepsilon_{m} \operatorname{Re}\left[\frac{\varepsilon_{p}^{*}-\varepsilon_{m}^{*}}{\varepsilon_{p}^{*}+2 \varepsilon_{m}^{*}}\right] \nabla\left|E^{2}\right| .
\end{gathered}
$$

Here $\varepsilon_{m}$ is the absolute dielectric constant of the medium; $\operatorname{Re}\left[F_{C M}\right]$ is the real part of the Clausius-Massotti factor, the sign of which determines the direction of the acting force, positive $(+)$ or negative $(-) \mathrm{DEPh}$, and $\varepsilon_{p}^{*}$ and $\varepsilon_{m}^{*}$ are complex dielectric constants of the particle and the medium, respectively. At $+\mathrm{DEPh}$ $\left(\varepsilon_{p}^{*}>\varepsilon_{m}^{*}\right)$, the particles move into the region of high fields and are captured in the space between the electrodes to which an alternating electric field is applied. At $-\operatorname{DEPh}\left(\varepsilon_{p}^{*}<\varepsilon_{m}^{*}\right)$, the particles are displaced from the area between the DEPh-electrodes.

Since the polarizability of bioparticles reflects their uniqueness and is determined by their shape/size (see expression (1)), the use of the DEPh effect opens up wide-ranging possibilities for manipulating the location of the analyte and background particles between the DEPh-electrodes. Currently, the successful use of DEPh for manipulating nanomaterials, Si-NWs [14], bioparticles, including their separation in solutions [13], self-formation of interfaces between NWs and bioparticles (bio/nanointerfaces) [15], etc., is well known.

The aim of this study is to use DEPh for a controlled change in the concentration of bioparticles in the region of the NW-sensor elements. For this purpose, SOI sensors with lateral $\mathrm{DEPh}$-electrodes were manufactured. The objectives of the study are to determine the response of sensors without DEPh and with $\mathrm{DEPh}$-controlled manipulation of viruses when exposed to DEPh of different signs. Nuclear polyhedrosis viruses and vaccinia viruses were used as an analyte. The research results showed the possibility of increasing the targeted delivery of the analyte to the sensor element due to the positive DEPh and the possibility of isolating the useful signal at the background level with a negative DEPh without using the surface modification of the sensors. The paper also discusses the advantages and limitations related to the use of $\mathrm{DEPh}$ for the indication of bioparticles.

\section{EXPERIMENTAL}

SOI sensors were made based on SOI films (Smart-Cut [16]) of $p$-type conductivity with the following design parameters: thickness of the cut-off silicon layer $t_{s i}=30 \mathrm{~nm}$ and buried oxide thickness $t_{b o x}=$ $200 \mathrm{~nm}$. The concentration of the acceptors in the SOI film was $2 \times 10^{16} \mathrm{~cm}^{-3}$. The sensor elements were SOI strips $1 \mu \mathrm{m}$ wide, $10 \mu \mathrm{m}$ long, having drain-source contact regions at the ends. Some of the on-chip sensors contained lateral DEPh-electrodes, which were made of highly doped CVD polysilicon. Since the maximum electric field strength is achieved in the corner regions of the rectangular electrodes, we used a design with offset (asymmetric) electrodes relative to each other in order to obtain the maximum value $E$ in the center of the sensor element. The optical and schematic image of the sensor is shown in Fig. 1. The chip's surface was insulated with a film of pyrolytic silicon dioxide. In the area of the sensor elements (central part in Fig. 1a), the protective layer hadwindows for the open access of solutions with the analyte.

The time dependences of the sensor current were measured $I_{\mathrm{si}}(t)$ with the sequential application of aqueous solutions of the analyte of different concentrations to their surface. The sensor current was recorded using a multichannel data acquisition system based on an NI 6363 module with a LabView graphical programming package (National Instrument, United States). In the measurements, the substrate of SOI structures was used as the control electrode of the bot- 
tom or back-gate (BG), which allows purposefully changing the conductivity mode of the sensor element (Fig. 1b). An easily programmable algorithm was used to select the voltage at the $\mathrm{BG}$, which provides the maximum response to the charge adsorbed on the surface of the sensors, which is described in detail in [17]. A Pt electrode, which maintains a constant potential of the solution on the surface of the sensors, was used as the reference electrode (RE). An inhomogeneous electric field in the space between the lateral electrodes and the sensor elements was created by an alternating voltage applied to the electrodes (gates) Z-1 and Z-2 (Fig. 1a). The sensors' response to the analyte's adsorption was defined as

$$
\text { Resp. }=\left(I_{\mathrm{ds}}-I_{\mathrm{ds}}^{\mathrm{o}}\right) / I_{\mathrm{ds}}^{\mathrm{o}} \text {. }
$$

Here $I_{\mathrm{ds}}^{\mathrm{o}}$ is the value of the sensor's current in deionized water before adding the analyte to the solution.

As an analyte, we used nuclear polyhedrosis viruses (NPVs) and vaccinia viruses (VVs). The initial viral suspensions were obtained at Rospotrebnadzor's Federal Budgetary Institution of Science, Vector State Research Center of Virology and Biotechnology and represented the following viruses:

(1) the NPV at a concentration of $10^{11} \mathrm{~mL}^{-1}$ (or $\left.\sim 2 \times 10^{-10} \mathrm{M}\right)$ in a $0.3 \mathrm{M}$ sucrose solution;

(2) the vaccinia virus inactivated by beta-propiolactone, strain LIVP at a concentration of $10^{9} \mathrm{~mL}^{-1}$ (or $\sim 2 \times 10^{-12} \mathrm{M}$ ). The initial solutions also contained residual proteins of the virus-supporting medium. In order to obtain isotonic solutions of highly resistant viruses, they were diluted in deionized water (DI). Solutions with different concentrations of viruses (samples) were applied onto the surface of the sensors by the drop method (pipetting). The droplet volume was $\sim 0.5 \mu \mathrm{L}$.

The viruses used in this work (and their complexes) are transparent in aqueous solutions. In order to determine the sign of DEPh for a transparent analyte in solutions, its DEPh-controlled motion was visualized in an optical microscope in transmission. For this, we used test structures in the form of rectangular chromium DEPh-electrodes deposited on glass. The distance between the electrodes was $70 \mu \mathrm{m}$.

\section{RESULTS AND DISCUSSION}

For a qualitative understanding of the features of the distribution of the electric field strength $E$ (without taking into account the effects related to the diffusion of mobile ions in the electrolyte, charge adsorption on the electrodes, etc.), scaled modeling of the investigated structures in the TCAD Sentarius environment was used. The asymmetric and symmetric configurations of DEPh-electrodes (with and without displacement relative to each other, respectively) are considered. In the simulation, the electrolyte was taken as a medium with a dielectric constant $\varepsilon_{m}=81$, and the voltage across electrodes Z-1 and Z-2 was taken equal to 8 and $0 \mathrm{~V}$, respectively.

Figure 2 shows the results of modeling the distribution of the electric field strength $E$ in structures with $\mathrm{DEPh}$-electrodes of two configurations and the corresponding $E^{2}(x)$ dependences obtained in different sections of the structures. As expected, the structures with displaced electrodes, in comparison with the symmetric configuration, provide the maximum values $E$ and maximum gradient $E^{2}$.

The results of modeling the distribution of the electric field strength $E$ for SOI sensors with asymmetric $\mathrm{DEPh}$-electrodes and $E^{2}(x)$ dependences obtained at different voltages at the control gate of the BG are shown in Fig. 3. The values $E^{2}$ have the following properties:

(1) They have maximums near the DEPh-electrode and NW-sensor element;

(2) They depend on the voltage $V_{\mathrm{BG}}$. An increase in the control voltage $V_{\mathrm{BG}}$ leads to an increase in the gradient $E^{2}$ near the sensor element and its decrease near the $\mathrm{DEPh}$-electrode.

Figure 4 shows an optical image of structures with symmetric electrodes during the DEPh manipulation of NPVs at a frequency of alternating voltage of $2 \times 10^{4} \mathrm{~Hz}$. The research results showed that a positive DEPh is observed in the subMHz range for the NPV. An increase in the concentration of viruses, first of all, occurs in the corners of the electrodes, i.e., in accordance with the data in Fig. 2, particles are collected in the area of increased values of $\nabla E^{2}$.

Figure 5 shows the time dependences of the current of sensors with +DEPh-control and without DEPhcontrol, measured with the indication of the NPV of a different concentration in the samples. For comparison, the original current values $I_{\mathrm{si}}$ of the sensors (in deionized water) are brought to the same level. It can be seen that the sensor without DEPh control reacts poorly to samples with a low analyte concentration. For a sensor with DEPh control, an increase is observed in the value of $I_{\mathrm{si}}$ with a delay of $100-200 \mathrm{~s}$ relative to the moment of introducing samples with the NPV. For samples with a subattomolar analyte concentration (units of $10^{-17} \mathrm{M}$ ), the response of sensors with DEPh- and without DEPh-control is (23-90) and $10 \%$, respectively. An increase in the sensor current is characteristic of the adsorption of positively charged particles on its surface; in the investigated solutions, the NPVs are positively charged.

Figure 6 shows the optical images of the sensors after the indication of the NPV, washing in the DW, and drying. Despite the fact that the surface of the sensors for covalent binding with the viruses was not modified (i.e., their physical adsorption is observed in the experiments), it can be seen from Fig. 6 that purification in DW does not completely remove the viruses 
(a)
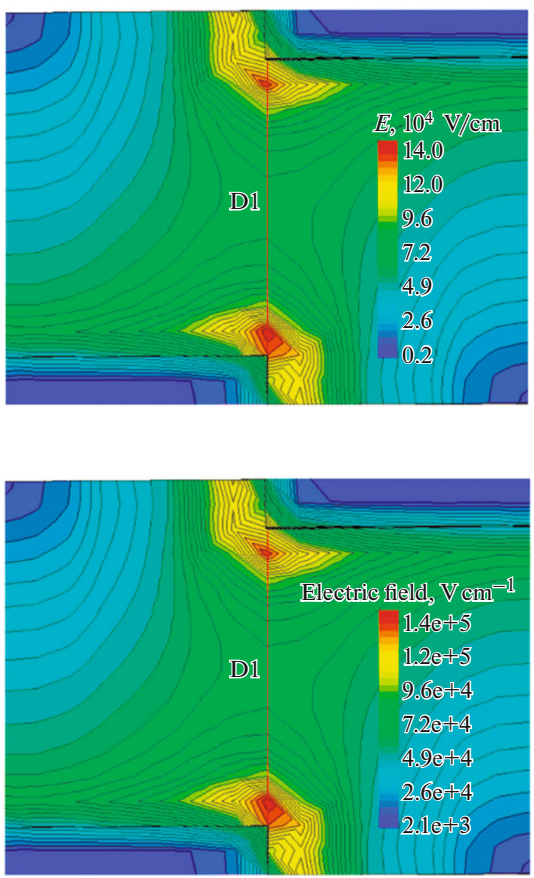

(b)
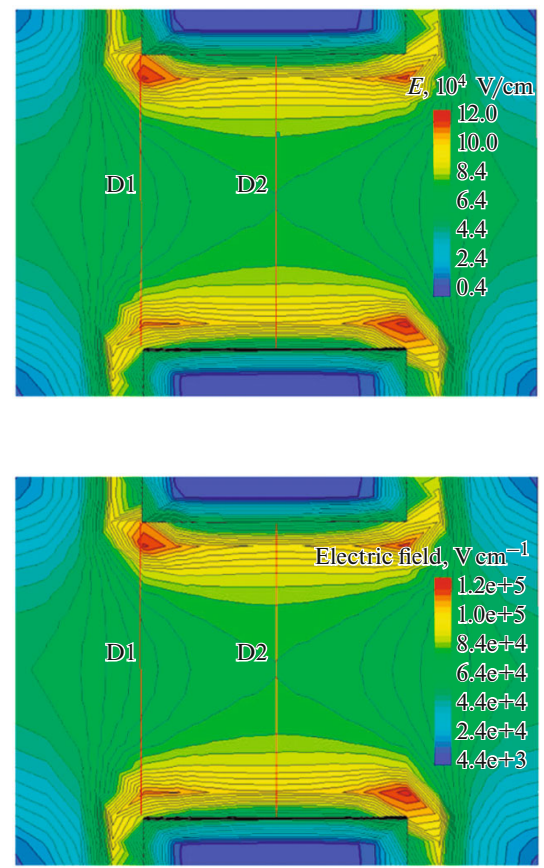

(c)

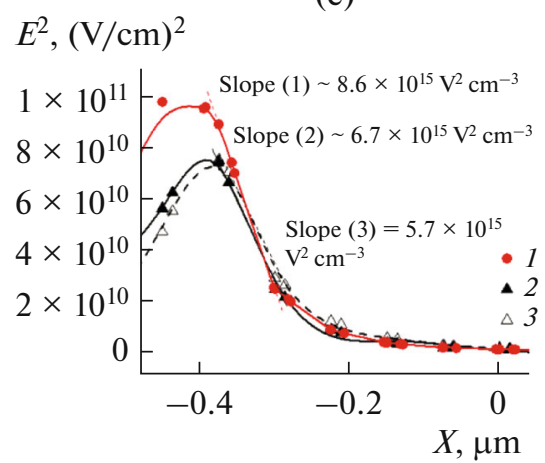

$E^{2},(\mathrm{~V} / \mathrm{cm})^{2}$

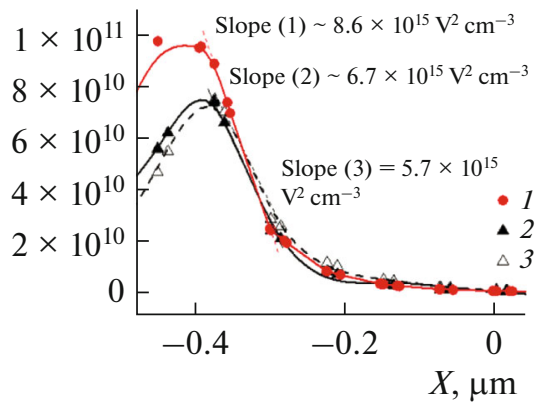

Fig. 2. Electric field strength distribution $E$ in structures with (a) asymmetric and (b) symmetric DEPh-electrodes, (c) $E^{2}(x)$ dependences for structures of type (a) 1 and (b) 2, 3 in sections $\mathrm{C} 1: 1,2$; and $\mathrm{C} 2,3 . x=0$ corresponds to half of the distance between the electrodes.

(a)

$E, 10^{4} \mathrm{~V} / \mathrm{cm}$

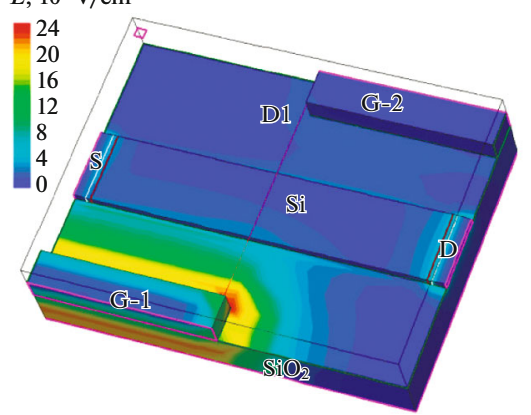

Electric field,

$\mathrm{V} \mathrm{cm}^{-1}$

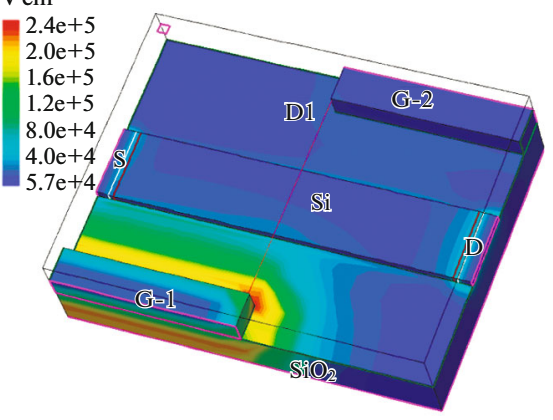

(b)

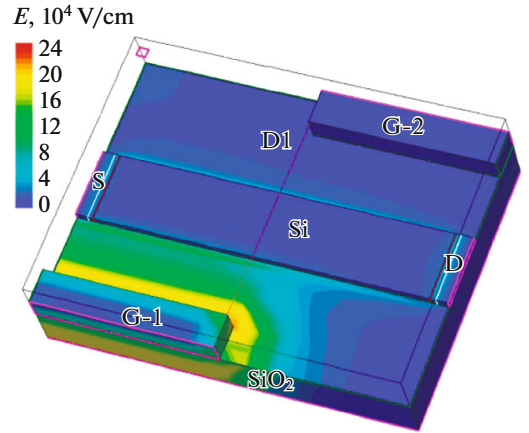

Electric field,

$\mathrm{V} \mathrm{cm}^{-1}$

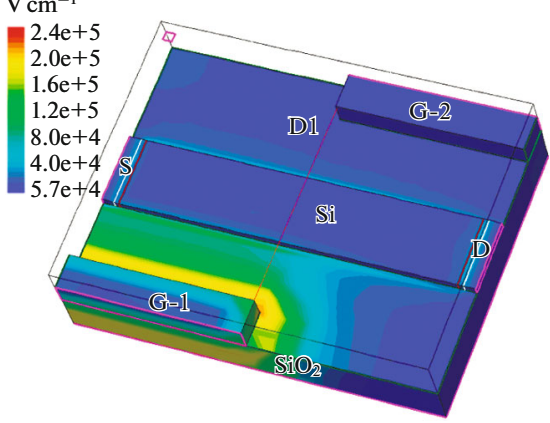

(c)

$E^{2},(\mathrm{~V} / \mathrm{cm})^{2}$

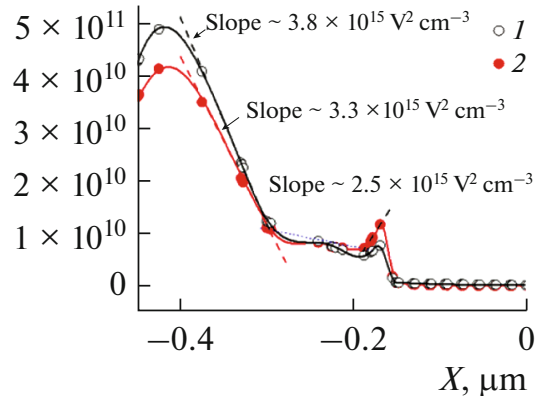

$E^{2},(\mathrm{~V} / \mathrm{cm})^{2}$

$5 \times 10^{11}$

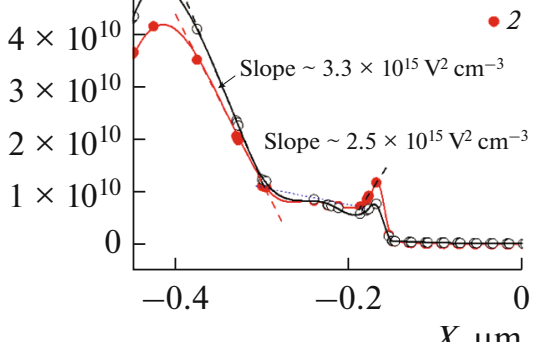

Fig. 3. The distribution of the electric field strength $E$ for a sensor with DEPh-electrodes at a voltage of $V_{\mathrm{RG}}, \mathrm{V}$ : (a) 0 and (b) 0.4 ; (c) $E^{2}(x)$ dependences at $V_{\mathrm{RG}}, \mathrm{V}:(1) 0$ and (2) 0.4 in section C1. $x=0$ corresponds to the middle of the sensor element. 
(a)

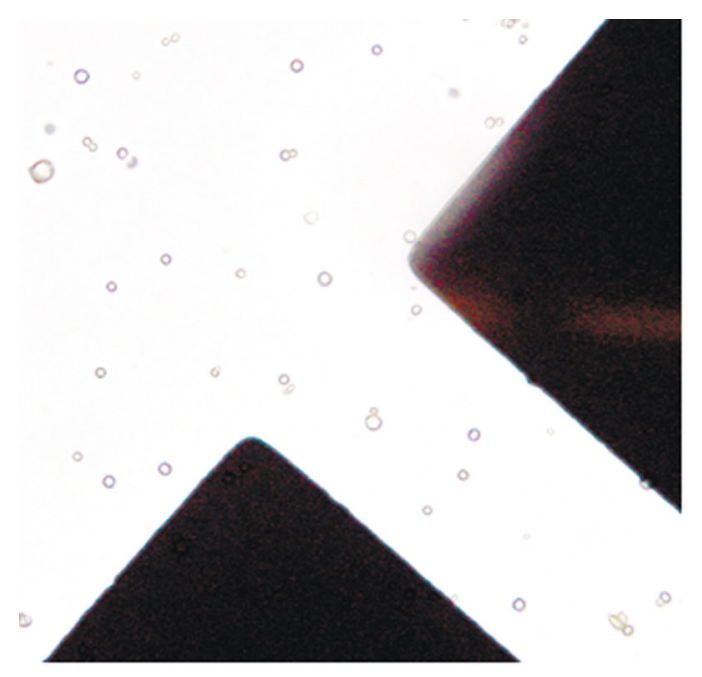

(b)

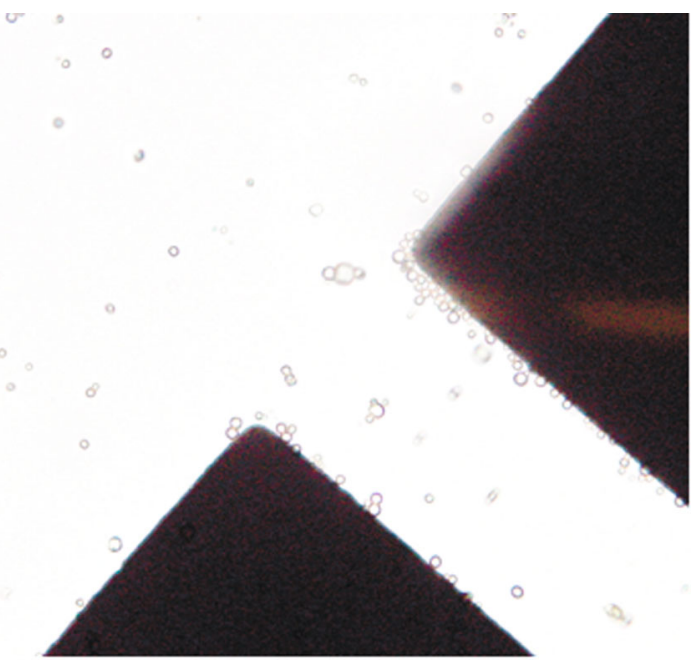

Fig. 4. Optical image of the distribution of the concentration of NPVs at a voltage on the DEPh-electrodes of $V_{\mathrm{s}}, \mathrm{V}: 0$ (a) and 8 (b). $f=2 \times 10^{4} \mathrm{~Hz}$.

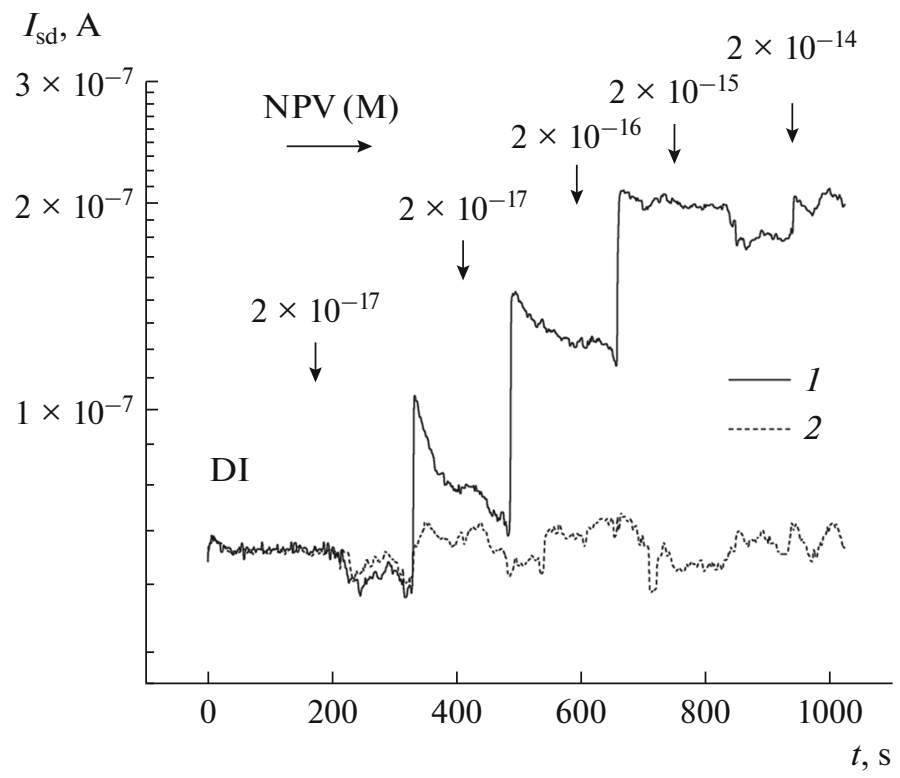

Fig. 5. $I_{\mathrm{si}}(t)$ dependence of sensors with DEPh-control ( 1 ) and without DEPh-control (2) during the indication of NPVs in samples with different concentrations of viruses. $f=2 \times 10^{4} \mathrm{~Hz}$.

from the crystal's surface. Sensors with DEPh-electrodes, unlike sensors without DEPh-electrodes, contain viruses on the surface. Thus, the use of $+\mathrm{DEPh}$ in the subMHz range makes it possible to increase the concentration of the NPVs in the area of the sensor element and, accordingly, its response.

The $I_{\mathrm{si}}(t)$ sensor dependences when testing samples with the vaccinia virus are shown in Fig. 7. It can be seen that with an increase in the VV concentration, in contrast to the NPV (compare with Fig. 5), a decrease in the current of the $I_{\mathrm{si}}$ sensors is observed. Moreover, the current modulation is greater for a sensor with DEPh control.

A decrease in the sensor current means an increase in the effective negative charge on its surface (more precisely, on and near the sensor surface in a solution within the Debye screening length), i.e., the effective charge of the analyte and background particles in the samples with $\mathrm{VV}$ is negative. The experiments on visualization of the DEPh-control of viruses showed that 
(a)

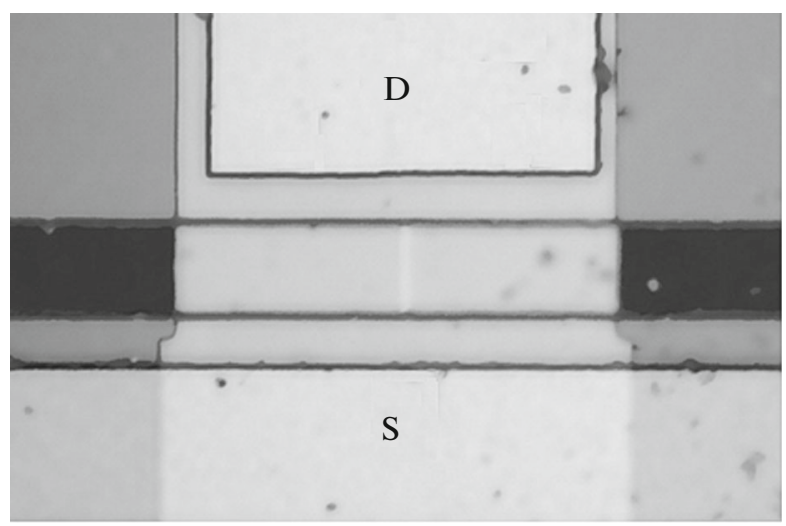

(b)

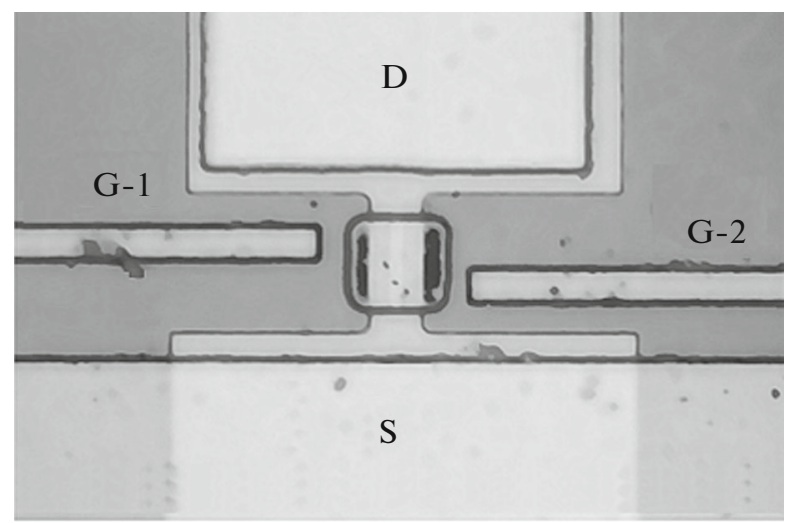

Fig. 6. Optical image of sensors: (a) without DEPh-electrodes; (b) with DEPh-electrodes after detection of the NPV and purification in DI.

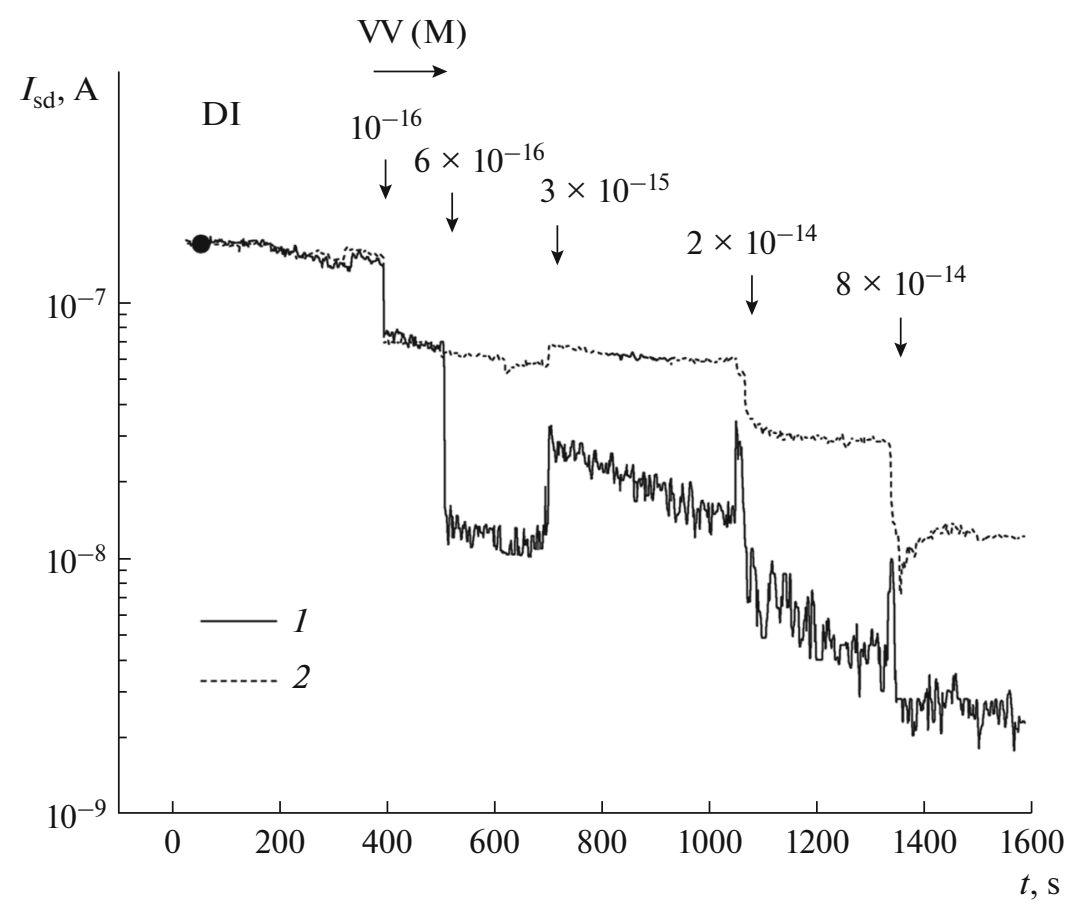

Fig. 7. $I_{\mathrm{si}}(t)$ dependence of sensors with $\mathrm{DEPh}$-control (1) and without DEPh-control (2) during the indication of vaccinia viruses in samples. $f=5 \times 10^{4} \mathrm{~Hz}$.

in the frequency range of $10^{4}$ to $10^{5} \mathrm{~Hz}$, there is a negative $\mathrm{DEPh}$ for $\mathrm{VV}$; i.e., the viruses are expelled from the area between the electrodes. Therefore, smaller values of $I_{\text {sd }}$ for sensors with DEPh-control, in comparison with sensors without DEPh-control, mean that at -DEPh there is a depletion of the area around the sensor element with positively charged vaccinia viruses.

Thus, the DEPh manipulation of the analyte can be used not only to increase the detectivity of sensors but also to verify the signal from the analyte and its charge state in solutions. At the same time, as the experiments carried out on viruses show, the surface of the sensors does not need to be modified. This approach has the following effects:

(1) it removes a number of problems related to the multistage modification of the surface of the sensors [11], including the problem of a possible change in the properties of the analyte itself during its interaction with probes;

(2) it provides the ability to create relatively inexpensive and highly sensitive test systems, including for 
laboratory research express diagnostics in the field of virology and microbiology, which is especially important, as is being shown with the the current pandemic.

Another positive aspect of using DEPh-controlled sensors is that their design parameters provide a certain selectivity in the indication of the analyte. The dielectrophoretic force, according to expression (1), depends on the geometric dimensions of the particles and the strength of the electric field (respectively, the distance between the electrodes). The effective DEPh control of viruses and bacteria requires a distance between the electrodes at the level of units and tens of microns; and for controlling proteins, a submicron range. In other words, in the case of sensors with $\mathrm{DEPh}$-controlled viruses and bacteria, the used alternating voltage does not affect the concentration of the background proteins near the sensor element.

The method has, among others, the following disadvantages. As the frequency increases, the electrolyte's conductivity increases (capacitive component of the sensor-electrolyte conductivity). This means limiting the frequency range when displaying the analyte in real time. For example, for aqueous solutions of the viruses studied in this paper, this limitation lies in the $\mathrm{MHz}$ region. Research has shown that for the vaccinia virus $+\mathrm{DEPh}$ is observed at a frequency of a few $\mathrm{MHz}$. In this case, the result of a $+D E P h$ increase in the $\mathrm{VV}$ concentration on the sensor element can be obtained only as follows:

(1) when immobilizing (fixing) the analyte on the sensor at high frequencies using the appropriate probes;

(2) in the subsequent measurement of the $I_{\mathrm{sd}}(t)$ dependences at the permissible, lower, frequencies; i.e., the process $+\mathrm{DEPh}$ manipulation and indications (measurement of $I_{\mathrm{si}}(t)$ dependences) must be spaced in time.

In addition, viruses, bacteria, etc., are bioparticles that can change their properties. For example, with prolonged exposure to an alternating signal, we observed a tendency for the formation of complex and a decrease, up to the complete loss, of the mobility of the viruses. In any case, DEPh-controlled sensors are only an instrumental platform with the option to control the analyte's concentration in the area of the sensor element. The test system must have a specific purpose and be optimized for a specific type of analyte.

\section{CONCLUSIONS}

The results obtained in this study show that SOI sensors with DEPh control electrodes made based on optical lithography have the following advantages:

(1) They can provide an express indication of viruses with a sensitivity of $\sim 2 \times 10^{-17} \mathrm{M}$;

(2) They can increase the response of the sensors by factors of 2 to 9 (compared to the response of sensors without DEPh control) at a subattomolar concentration of the analyte;

(3) They can determine the charge state of viruses (in particular, the positive effective charge of the NPV and VV) in the tested solutions without modifying the surface of the sensors.

Outlined in this work, results of the use of highly sensitive SOI sensors for recording viruses carrying socially significant diseases, together with the data accumulated on their use in solving various problems of biomedicine, illustrate the promising prospects of their use as an instrumental platform for research and diagnostic tasks for the indication of pathogens of viral and bacterial forms in real time, which is extremely important in emergency (epidemic, pandemic, etc.) situations.

\section{ACKNOWLEDGMENTS}

The authors thank Boris Ivanovich Fomin, the leading researcher at Rzhanov Institute of Semiconductor Physics, Siberian Branch, Russian Academy of Sciences (ISP SB RAS), who has died untimely, and the main developer of the SOI sensor technology, without whose contribution none of the listed works of the authors, including this one, would have appeared. The authors thank Doctor of Physics D.V. Shcheglova for discussing and assisting in organizing the study.

\section{FUNDING}

This study was supported by grant no. 18-29-02091 of the Russian Foundation for Basic Research.

The virus samples were prepared as part of state task GZ $11 / 16$ of the sectoral research program of Rospotrebnadzor for 2016-2020 "Problem-oriented research in the field of the epidemiological surveillance of infectious and parasitic diseases."

\section{REFERENCES}

1. Neizvestnyi, I.G., Semiconductor nanowire sensors, Russ. Microelectron., 2009, vol. 38, no. 4, pp. 223-238.

2. Naumova, O.V., Popov, V.P., Safronov, L.N., Fomin, B.I., Nasimov, D.A., Latyshev, A.V., Aseev, A.L., Ivanov, Yu.D., and Archakov, A.I., Ultra-thin SOI layer nanostructuring and nanowire transistor formation for femtomole electronic biosensors, ESC Trans., 2009, vol. 25 , no. 10 , pp. 83-87.

3. Naumova, O.V., Fomin, B.I., Safronov, L.N., Nasimov, D.A., Il'nitskii, M.A., Dudchenko, N.V., Devyatova, S.F., Zhanaev, E.D., Popov, V.P., Latyshev, A.V., and Aseev, A.L., Silicon nanowire transistors for electron biosensors, Optoelectron., Instrum. Data Process., 2009, vol. 45, no. 4, pp. 287-291.

4. Naumova, O.V., Fomin, B.I., Nasimov, D.A., Dudchenko, N.V., Devyatova, S.F., Zhanaev, E.D., Popov, V.P., Latyshev, A.V., Aseev, A.L., Ivanov, Yu.D., and Archakov, A.I., SOI nanowires as sensors for 
charge detection, Semicond. Sci. Technol., 2010, vol. 25, p. 055004.

5. Ivanov, Yu.D., Pleshakova, T.O., Kozlov, A.F., Malsagova, K.A., Krohin, N.V., Shumyantseva, V.V., Shumov, I.D., Popov, V.P., Naumova, O.V., Fomin, B.I., Nasimov, D.A., Aseev, A.L., and Archakov, A.I., SOI nanowire for the high-sensitive detection of HBsAg and $\alpha$-fetoprotein, Lab on a Chip, 2012, vol. 12, no. 23, pp. 5104-5111.

6. Ivanov, Yu.D., Pleshakova, T.O., Kozlov, A.F., Mal'sagova, K.A., Krokhin, N.V., Kaisheva, A.L., Shumov, I.D., Popov, V.P., Naumova, O.V., Fomin, B.I., Nasimov, D.A., Aseev, A.L., and Archakov, A.I., SOI nanowire transistor for detection of D-NFATc1 molecules, Optoelectron., Instrum. Data Process., 2013, vol. 49, no. 5, p. 520.

7. Patolsky, F., Zheng, G.F., Hayden, O., Lakadamyali, M., Zhuang, X.W., and Lieber, C.M., Electrical detection of single viruses, Proc. Natl. Acad. Sci. U. S. A., 2004, vol. 101, pp. 14017-14022.

8. Dmitrienko, E., Naumova, O., Fomin, B., Kupryushkin, M., Volkova, A., Amirkhanov, N., Semenov, D., Pyshnaya, I., and Pyshnyi, D., Surface modification of SOI-FET sensors for label-free and specific detection of short RNA analyte, Nanomedicine, 2016, vol. 11, no. 16, pp. 2073-2082.

9. Ivanov, Yu.D., Pleshakova, T.O., Malsagova, K.A., Kozlov, A.F., Kaysheva, A.L., Shumov, I.D., Gabiullin, R.A., Kurbatov, L.V., Popov, V.P., Naumova, O.V., Fomin, B.I., Nasimov, D.A., Aseev, A.L., Alferov, A.A., Kushlinsky, N.E., Lisitsa, A.V., and Archakov, A.I., Detection of marker miRNAs in plasma using SOI-NW biosensor, Sens. Actuators, B, 2018, vol. 261, pp. 566571 .
10. Generalov, V.M., Naumova, O.V., Fomin, B.I., P'yankov, S.A., Khlistun, I.V., Safatov, A.S., Zaitsev, B.N., Zaitseva, E.G., and Aseev, A.L., Detection of Ebola virus VP40 protein using a nanowire SOI biosensor, $O p$ toelectron., Instrum. Data Process., 2019, vol. 55, no. 6, pp. 618-622.

11. Naumova, O.V., Fomin, B.I., Dmitrienko, E.V., Pyshnaya, I.A., and Pyshnyi, D.V., Surface modification of SOI sensors for the detection of RNA biomarkers, Semiconductors, 2020, vol. 54, no. 4, pp. 471-475.

12. Naumova, O.V., Fomin, B.I., Malyarenko, N.F., and Popov, V.P., Modification and characterization of the surface of SOI nanowire sensors, J. Nano Res., 2012, vols. $18-19$, pp. 139-147.

13. Qian, C., Huang, H., Chen, Liguo, Li, X., Ge, Z., Chen, T., Yang, Z., and Sun, L., Dielectrophoresis for bioparticle manipulation, Int. J. Mol. Sci., 2014, vol. 15, pp. 18281-18309.

14. Santos, M.V.P., Béron, F., Pirota, K.R., Diniz, J.A., and Moshkalev, S., Electrical manipulation of a single nanowire by dielectrophoresis, in Nanowires-New Insights, Rijeka: InTech Open, 2017, chap. 3, pp. 41-58. https://doi.org/10.5772/67386

15. Suehiro, J., Fabrication and characterization of nanomaterial-based sensors using dielectrophoresis, Biomicrofluidics, 2010, vol. 4, p. 022804.

16. Celler, G.K. and Cristoloveanu, S., Frontiers of silicon-on-insulator, J. Appl. Phys., 2003, vol. 93, no. 9, pp. 4955-4978.

17. Naumova, O.V. and Fomin, B.I., Optimization of the response of nanowire biosensors, Optoelectron., Instrum. Data Process., 2016, vol. 52, no. 5, pp. 434-437. 